\title{
On the nature of the break in the X-ray luminosity function of low-mass X-ray binaries
}

\author{
M. Revnivtsev ${ }^{1,2}$, K. Postnov ${ }^{3}$, A. Kuranov ${ }^{3}$, and H. Ritter ${ }^{4}$ \\ ${ }^{1}$ Excellence Cluster Universe, Technische Universität München, Boltzmannstr. 2, 85748 Garching, Germany \\ e-mail: mikej@mpa-garching.mpg.de \\ 2 Space Research Institute, Russian Academy of Sciences, Profsoyuznaya 84/32, 117997 Moscow, Russia \\ 3 Sternberg Astronomical Institute, Moscow State University, Universitetskij pr., 13, 119992 Moscow, Russia \\ ${ }^{4}$ Max-Planck-Institute für Astrophysik, Karl-Schwarzschild-Str. 1, 85740 Garching bei München, Germany \\ Received 2 April 2010 / Accepted 18 November 2010
}

\section{ABSTRACT}

\begin{abstract}
We analyze a flux-limited sample of persistent and bright (with 2-10 keV fluxes exceeding $1.4 \times 10^{-10} \mathrm{erg} \mathrm{s}^{-1} \mathrm{~cm}^{-2}$ ) low-mass X-ray binaries (LMXBs) in our Galaxy. It is demonstrated that the majority of binary systems with X-ray luminosities below $\log L(\mathrm{erg} / \mathrm{s}) \sim$ 37.3 have unevolved secondary companions (except for those with white dwarf donors), while systems with higher X-ray luminosity harbor predominantly giant donors. Mass transfer in binary systems with giants significantly shortens their lifetime thus steepening the X-ray luminosity function of LMXBs at high luminosity. We argue that this is why the LMXB luminosity function constructed from observations of sources in our and distant galaxies demonstrates a break at $\log L(\mathrm{erg} / \mathrm{s}) \sim 37.3$.
\end{abstract}

Key words. binaries: general - stars: low-mass - stars: luminosity function, mass function - stars: neutron - X-rays: stars

\section{Introduction}

Binary systems with low-mass secondary companions to a compact star (black hole or neutron star) were discovered in the 1960s at the dawn of X-ray astronomy. All-sky surveys performed by different orbital X-ray observatories (UHURU, HEAO1, Ariel V, etc.) provided us with a relatively large sample of these objects in our Galaxy. The advent of focusing $\mathrm{X}$-ray telescopes with an angular resolution of arcseconds initiated studies of these low-mass X-ray binaries (LMXBs), first in nearby galaxies, such as M 31 (Trinchieri \& Fabbiano 1991; Primini et al. 1993), and then in more distant galaxies (see e.g. reviews in Kim \& Fabbiano 2004; Gilfanov 2004).

The accretion luminosity $L_{\mathrm{x}}$ of a persistent (sub-Eddington) LMXB is directly proportional to the mass transfer rate $\dot{M}$ from the secondary star. According to the standard theory of binary star evolution, the persistent mass transfer via the inner Lagrangian point in a close binary is due to an increase in the size of the donor star relative to its Roche lobe. In an LMXB, this can be done either by decreasing the size of the Roche lobe by the loss of orbital angular momentum (see e.g. Paczynski \& Sienkiewicz 1981; Verbunt \& Zwaan 1981), or increasing the radius of the star as a consequence of its nuclear evolution (Webbink et al. 1983; Taam 1983; Ritter 1999). The observed properties of different populations of LMXBs can be used to test the models of binary evolution.

The X-ray luminosity function (LF) is an important characteristic of the LMXB population. In other galaxies, it is compiled directly from measured X-ray fluxes down to a luminosity $L_{\mathrm{x}} \sim 10^{37} \mathrm{erg} / \mathrm{s}$ and can be fitted by a power law $\mathrm{d} N / \mathrm{d} \log L \propto L^{-0.8 \ldots-1.2}$ with a steepening at luminosities exceeding the Eddington limit for accreting neutron stars $\left(\log L_{\mathrm{x}}>\right.$ 38.5) (Kim \& Fabbiano 2004). However, it has become possible to construct the luminosity functions of LMXBs in nearby galaxies, such as M 31 or Cen A, down to much fainter luminosities of the order of $10^{36} \mathrm{erg} / \mathrm{s}$ (Primini et al. 1993; Gilfanov 2004; Voss \& Gilfanov 2006, 2007; Voss et al. 2009). Over such a wide luminosity interval, the luminosity function of LMXBs can no longer be described by a single power law and has a characteristic break at $\log L<37.3$ (Gilfanov 2004). A similar result down to luminosities $L_{\mathrm{x}} \sim 10^{35} \mathrm{erg} / \mathrm{s}$ was obtained for Galactic LMXBs from all-sky surveys (Grimm et al. 2002; Gilfanov 2004) and the survey of the Galactic bulge (Revnivtsev et al. 2008). The statistical significance of the LF break in the above-mentioned works is high, therefore the existence of the break requires physical explaination. One of the clearest cases of the flattening of the LMXB LF at low luminosities can be seen in the work of Revnivtsev et al. (2008), where the LMXB candidates in the bulge of our Galaxy were traced down to luminosities $10^{35} \mathrm{erg} / \mathrm{s}$, almost unreachable for LMXBs in outer galaxies.

Different explanations have been proposed for the origin of the break in the observed LF of LMXBs. For example, the break in the LF might be caused by the change in the dominant orbital angular momentum loss mechanism from magnetic stellar wind (Verbunt \& Zwaan 1981) to gravitational wave emission (see e.g. Paczynski \& Sienkiewicz 1981) in the population of LMXBs with low-mass main sequence secondaries (Postnov \& Kuranov 2005). It has also been noted that the most luminous LMXBs with high mass transfer rates $\left(\dot{M}>10^{-8} M_{\odot} /\right.$ year $)$ could harbor giant donors (see e.g. Webbink et al. 1983), which may explain the difference between the low- and high-luminosity sources. The study of the LMXB LF in galaxies using population synthesis methods could potentially be quite powerful (see e.g. Fragos et al. 2008; Kim et al. 2009). However, this approach involves a number of uncertain parameters of binary star evolution, so it is difficult to make firm conclusions based on the population synthesis simulations alone. 
In this paper, we attempt to identify the main physical reason for the origin of the observed break of the broad-band luminosity function of LMXBs.

We show that the break in the LF of LMXBs is probably caused by different types of donor stars: evolved secondaries (giants) at luminosities above the break at $\log L(\mathrm{erg} / \mathrm{s}) \sim 37.3$, and main-sequence stars at lower luminosities. The correctness of our conclusion might be checked by direct calculations of the LMXB properties using the methods of population synthesis, which we plan to do in the future.

\section{A flux-limited sample of persistent LMXBs in the Galaxy}

We consider only persistent LMXBs. This selection can be relatively easily done for Galactic LMXBs, but in general this can be a difficult task for LMXBs in outer galaxies, since they are typically observed only during short time intervals. Monitoring $\mathrm{X}$-ray sources in our Galaxy shows that at any particular time there are only a few bright transients (see e.g. Remillard et al. 2009), whose effect on the instantaneous LF of all Galactic LMXBs is rather small.

Moreover, the properties of the observed luminosity distribution of individual variable LMXBs were found not to affect the shape of the instantaneous LF of the Galactic sample (Postnov $\&$ Kuranov 2005). Therefore the results of our present study can also be applied to the instantaneous sample of LMXBs in distant galaxies.

We study a representative sample of persistent Galactic LMXBs with X-ray luminosities $L_{\mathrm{x}}>10^{36} \mathrm{erg} / \mathrm{s}$ and compare their properties above and below the break. Our goal does not require the sample to be either volume limited or complete, because we are not interested in statistics (which has been extensively studied in works of other authors), but rather searching for a correspondence of the time-averaged X-ray luminosity of sources to some parameters of their binaries.

To select persistent Galactic sources, we can analyze any existing X-ray survey. However, the chosen survey should fulfil the following requirements: 1) it should cover as much as possible of the Galactic plane region, where the majority of the LMXBs are located; 2) it should have an angular resolution of better than $\sim 2-3^{\circ}$ to avoid confusion within the Galactic plane; and 3) it should be performed in the energy band $\sim 1-20 \mathrm{keV}$ where Galactic LMXBs emit most of their bolometric luminosity. These requirements significantly shorten the list of usable surveys. In particular, the sky survey of the RXTE observatory (Revnivtsev et al. 2004) does not cover the region of the Galactic plane, and the scans of the RXTE observatory over the Galactic bulge and the Galactic plane (Markwardt et al. 2000) have not (yet) been used to perform a sky survey, but rather to measure the fluxes of preselected list of sources. Surveys carried out by the ASCA (Sugizaki et al. 2001; Sakano et al. 2002) and BeppoSAX (Sidoli et al. 2001) observatories cover only a small fraction of the Galaxy. Even smaller sky areas are covered by the Chandra and XMM observatories. The survey of the INTEGRAL observatory (Krivonos et al. 2007) covers the whole Galaxy, but was performed at hard X-ray energies, and is not fully suitable for our purposes (however, we use it for additional checks of "persistency" of selected sources).

Hence, the persistent X-ray sources detected by UHURU (Forman et al. 1978) turn out to be most suitable for our study; as an additional check of their persistent behavior we have also examined their presence in the INTEGRAL all-sky survey
(Krivonos et al. 2007). We selected only sources with $2-10 \mathrm{keV}$ fluxes above $1.4 \times 10^{-10} \mathrm{erg} \mathrm{s}^{-1} \mathrm{~cm}^{-2}$, which ensures that their luminosity is no fainter than $2-3 \times 10^{36} \mathrm{erg} / \mathrm{s}$ to distances of $12 \mathrm{kpc}$ (i.e. to the furthest edge of the Galactic bulge). This allows us to conclude that we do not miss brighter sources at least in more than a half of the Galaxy. We also included in our sample the black-hole binary GRS 1915+105, which was not seen by UHURU during its operation time (1971-1973), but has remained persistently bright since its appearance in 1992 (Castro-Tirado et al. 1992), and excluded the source GX 1+4, which is known to be accreting via a stellar wind (Hinkle et al. 2006) and not a Roche lobe overflow, which we consider here. The fluxes assigned to GRS 1915+105 and GS 1826-24 were taken from measurements of the All Sky Monitor of the RXTE observatory averaged over 1996-2009 ${ }^{1}$.

Energy fluxes from the selected sources were calculated from observed UHURU count rates, assuming that the Crab nebula count rate in the energy band $2-10 \mathrm{keV}$ corresponds to the energy flux $2.22 \times 10^{-8} \mathrm{erg} \mathrm{s}^{-1} \mathrm{~cm}^{-2}$.

In Table 1, we present the list of sources with values of their orbital periods and estimates of their distances and luminosities.

\section{Results}

From Table 1 and Fig. 1, it is clearly seen that all systems with X-ray luminosities exceeding $\sim 5 \times 10^{37} \mathrm{erg} / \mathrm{s}$ for which we have information about their orbital periods, apparently have large sizes. This (in combination with the assumption that the companion star fills its Roche lobe) means that donor stars in these systems must have large radii and are thus evolved stars (subgiants or giants). In several cases, this was shown directly from the observed spectra of the optical counterparts (Sco X-1, Bandyopadhyay et al. 1997; GX 349+2, Bandyopadhyay et al. 1999; Cyg X-2, Casares et al. 1998; GX 5-1, Bandyopadhyay et al. 2003; GRS 1915+105, Greiner et al. 2001; Cir X-1, Jonker et al. 2007).

In contrast, there are virtually no persistent LMXBs with $\mathrm{X}$-ray luminosities fainter than $\sim 10^{37.5} \mathrm{erg} / \mathrm{s}$ and orbital periods longer than 5-10 h (see also Ritter \& Kolb 2003; Liu et al. 2007). This is consistent with the assumption that donor stars in these systems are main-sequence stars (or degenerate dwarfs, if the orbital period is shorter than $\sim 1 \mathrm{~h}$ ). For cataclysmic variables with accreting white dwarfs and orbital periods shorter than $\sim 10 \mathrm{~h}$ (which are much more numerous in the Galaxy and can be found closer to the Sun, hence studied in much greater detail) this statement has been solidly supported by observations (Smith \& Dhillon 1998). Therefore, we conclude from observations that at luminosities $L_{\mathrm{x}} \sim 10^{37.5} \mathrm{erg} / \mathrm{s}$ there is a transition from LMXBs with predominantly giant donors to binaries with predominantly main-sequence donors.

\section{A simple model of the LMXB luminosity function}

Numerous studies have been made in the past to describe the evolution of the LMXB population using specific models of LMXB evolution. They were mostly aimed at understanding the evolution of the total number of Galactic LMXBs without distinguishing their X-ray luminosities (see e.g. White \& Ghosh 1998; Ghosh \& White 2001). Attempts to describe the LMXB LF can be found in Webbink et al. (1983), but this work concentrated only on the bright end of the LF and did not consider the effects of magnetic braking on the period/luminosity distribution

1 http://xte.mit.edu/asmlc/ASM.html 
Table 1. The brightest persistent low mass X-ray binaries in the Galaxy with fluxes $>1.4 \times 10^{-10} \mathrm{erg} \mathrm{s}^{-1} \mathrm{~cm}^{-2}$ measured by UHURU (Forman et al. 1978).

\begin{tabular}{|c|c|c|c|}
\hline Name & $\begin{array}{c}L_{\mathrm{x}}(2-10 \mathrm{keV}) \\
10^{37} \mathrm{erg} / \mathrm{s}\end{array}$ & Dist, kpc & Period, $\mathrm{h}$ \\
\hline Sco X-1 & 37.4 & $2.8^{1}$ & 18.94 \\
\hline GX 5-1 & 26.1 & $9^{2}$ & \\
\hline GRS $1915+105$ & 26.0 & $11^{3}$ & 739.20 \\
\hline Cyg X-2 & 19.4 & $11.6^{5}$ & 236.27 \\
\hline GX340+0 & 18.2 & $12^{4}$ & \\
\hline GX $349+2$ & 15.3 & $8.5^{6}$ & 22.5 \\
\hline GX $17+2$ & 14.9 & $7.5^{7}$ & \\
\hline GX 9+1 & 8.7 & $7.2^{7}$ & \\
\hline 4U1820-30 & 5.7 & $8^{8}$ & 0.19 \\
\hline Ser X-1 & 5.5 & $8.4^{9}$ & 12.96 \\
\hline GX $13+1$ & 5.5 & $6.9^{10}$ & 601.7 \\
\hline 4U1735-44 & 4.9 & $9.1^{11}$ & 4.65 \\
\hline GX 3+1 & 3.4 & $4.5^{12}$ & \\
\hline 4U1624-49 & 3.1 & $15^{13}$ & 20.9 \\
\hline 4U1636-53 & 2.4 & $5.9^{10}$ & 3.79 \\
\hline GX 9+9 & 1.8 & $5.0^{7}$ & 4.20 \\
\hline 4U1746-37 & 1.4 & $11.0^{8}$ & 5.16 \\
\hline $4 U 1705-32$ & 1.18 & $13^{14}$ & \\
\hline 1A1742-294 & 0.81 & $8.5^{15}$ & \\
\hline 4U1254-69 & 0.79 & $11^{16}$ & 3.93 \\
\hline 4U0513-40 & 0.74 & $12.1^{8}$ & 0.28 \\
\hline 4U1823-00 & 0.61 & $6.3^{17}$ & 3.19 \\
\hline 4U1915-05 & 0.43 & $8.8^{8}$ & 0.83 \\
\hline 4U0614+09 & 0.34 & $3.2^{18}$ & 0.81 \\
\hline 4U1702-42 & 0.32 & $6.2^{19}$ & \\
\hline $4 U 1626-67$ & 0.32 & $8^{20}$ & 0.69 \\
\hline GS1826-24 & 0.31 & $6.0^{21}$ & $2.25^{22}$ \\
\hline 4U1708-40 & 0.27 & $8^{15}$ & \\
\hline 4U1543-62 & 0.26 & $7.0^{8}$ & 0.30 \\
\hline $4 \mathrm{U} 1724-30$ & 0.17 & $9.5^{8}$ & \\
\hline 4U1850-08 & 0.17 & $8.2^{8}$ & 0.34 \\
\hline $4 \mathrm{U} 1812-12$ & 0.09 & $4.0^{19}$ & \\
\hline $4 \mathrm{U} 1556-60$ & 0.09 & $4.0^{7}$ & \\
\hline 4U1822-37 & $0.04^{*}$ & $2.5^{23}$ & 5.57 \\
\hline
\end{tabular}

Notes. Orbital periods of systems are adopted from Ritter \& Kolb (2003) and Liu et al. (2007).

* - The source belongs to a group of so called accretion disk corona sources, in which we do not see the direct emission of the innermost parts of the accretion flow, therefore the observed X-ray luminosity of such a source is only a part of its intrinsic X-ray luminosity.

References. (1) - Bradshaw et al. (1999); (2) - Jonker et al. (2000); (3) - Fender et al. (1999); (4) - van Paradijs \& White (1995); (5) Smale (1998); (6) - Wachter \& Margon (1996); (7) - Christian \& Swank (1997); (8) - Kuulkers et al. (2003); (9) - Ebisuzaki et al. (1984); (10) - Bandyopadhyay et al. (1999); (11) - Augusteijn et al. (1998); (12) - Kuulkers \& van der Klis (2000); (13) - Xiang et al. (2007); (14) - in't Zand et al. (2005); (15) - adopting distance to the Galactic Center; (16) - Courvoisier et al. (1986); (17) - Shahbaz et al. (2007); (18) - Kuulkers et al. (2010); (19) - Jonker \& Nelemans (2004); (20) - Chakrabarty (1998); (21) - Heger et al. (2007); (22) - Mescheryakov et al. (2010); (23)- Mason \& Cordova (1982).

of binaries. The importance of magnetic braking for relatively short period systems was emphasized, for example, by Pylyser \& Savonije (1988) and Cote \& Pylyser (1989). In these papers, an indication of a transition from a population of bright systems dominated by nuclear evolution of donor stars to a population of systems in which mass transfer is sustained by the magnetic braking can be found. At that time, it was impossible to compare quantitatively the model predictions with the observed samples

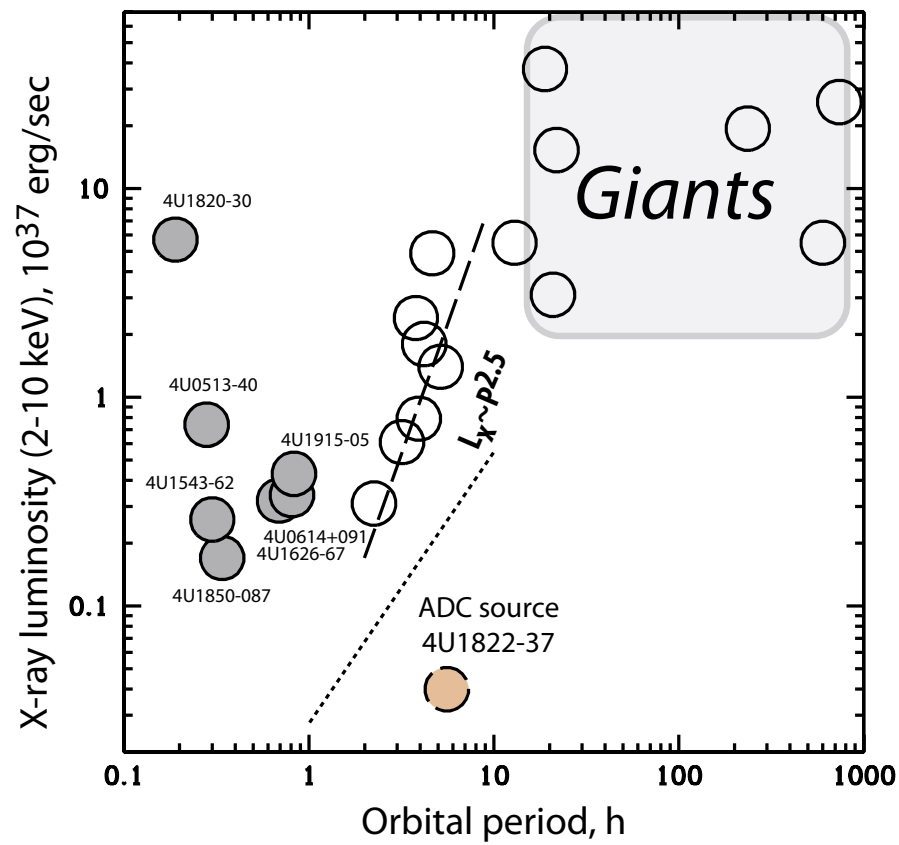

Fig. 1. Positions of the LMXBs from Table 1 on the orbital period $\mathrm{X}$-ray luminosity diagram. Filled circles denote the positions of peculiar systems: grey circles show the positions of binaries with white dwarf or hydrogen-deficit accretors, and the dashed circle shows the position of 4U1822-37 in which we see only a fraction of the total X-ray luminosity due to its nearly edge-on orientation (a so-called accretion disk corona source). The region, occupied by binary systems with a giant donor is shown by the shaded square. The dashed line shows the slope $L_{\mathrm{x}} \propto P^{2.5}$ derived by Iben \& Tutukov (1984) for the mass transfer rate in binaries in which angular momentum loss is driven by a magnetic stellar wind from a main-sequence donor. The dotted line shows the boundary of persistency according to the thermal-viscous disk instability for binaries with low-mass main-sequence donors $L_{\mathrm{x}} / 10^{37} \mathrm{erg} / \mathrm{s} \sim 0.025 P_{\mathrm{h}}^{1.4}$ from Eq. (32) of Dubus et al. (1999), where we adopted $M_{1}=1.4 M_{\odot}, M_{2}=$ $0.4 M_{\odot}$, and $L_{\mathrm{x}}=0.1 \dot{M} c^{2}$.

of LMXBs because the lack of sensitive Galactic X-ray surveys. Thus these studies were focused mostly on the exploration of evolutionary tracks leading to the formation of particular objects, such as rotation-powered pulsars (e.g. Kulkarni \& Narayan 1988; Naylor \& Podsiadlowski 1993; Deloye 2008, and references therein), or ultracompact binaries (e.g. Nelemans et al. 2001; Belczynski et al. 2002).

Many subsequent papers were devoted to the population synthesis of LMXBs (see e.g. Belczynski et al. 2002; Pfahl et al. 2003; van der Sluys et al. 2005; Fragos et al. 2008), which has been affected by substantial uncertainties in the binary star evolution (e.g. the treatment of the common envelope stage, parameters of magnetic braking, etc.). These detailed studies are clearly very important and must continue as they may help us to constrain the values of specific parameters of binary evolution by comparison with observations. However, to understand the observed gross properties of the LMXB LF we can try to adopt very general assumptions about binary star evolution, as we show below.

We consider an ensemble of binaries that formed in a galactic halo over a time period $T$ in the past. We define $\tau(P)$ to be the duration of an active LMXB stage of a source within the orbital period range $P, P+\mathrm{d} P$, and $n(P)$ to be the number distribution of sources within this period range. Assuming the steady formation 
of sources over time period $T$, the luminosity distribution can be written in the form

$$
\frac{\mathrm{d} N}{\mathrm{~d} L_{\mathrm{x}}} \propto \frac{\tau(P)}{T} n(P) \frac{\mathrm{d} P}{\mathrm{~d} L_{\mathrm{x}}}
$$

(We note that the first factor $\tau / T$ would not appear for a LF constructed from a sample of sources produced in an instantaneous star formation burst; however, this is the case for neither the Galactic bulge nor the elliptical galaxies we consider here.)

The shape of LF is clearly determined by the dependence of all three factors in this expression on the X-ray luminosity. We consider these factors separately for bright (with giant donors) and dim (with main-sequence donors) LMXBs.

At luminosities fainter $L_{\mathrm{x}} \approx 10^{37} \mathrm{erg} / \mathrm{s}\left(\dot{M}<10^{-9} M_{\odot} /\right.$ year $)$, the majority of donor stars in our LMXB sample are mainsequence stars with long lifetimes ( $>$ Gyr). The stable masstransfer episode is also very long, so the factor $\tau / T$ should not depend strongly on $L_{\mathrm{x}}$. The period distribution $n(P)$ is generally determined by the evolutionary history of binaries, which includes as a minimum a supernova explosion to produce the neutron star, a common envelope phase, etc. (or involves dynamical processes in dense stellar clusters), and can be found for example from population synthesis studies. We define it to be in the form $\mathrm{d} N / \mathrm{d} \log P$ and leave it as it is for a while.

The luminosity of a LMXB due to magnetic stellar wind braking depends on the orbital period in the form of a power law $L_{\mathrm{x}} \propto P^{2.5}$ (see e.g. Iben \& Tutukov 1984), $L_{\mathrm{x}} \propto P^{3.3}$ (Patterson $1984)$, or $L_{\mathrm{x}} \propto P^{3.8}$ (Cote \& Pylyser 1989), which written in the form $\mathrm{d} \log P / \mathrm{d} \log L_{\mathrm{x}}$ is independent of $L_{\mathrm{x}}$. Hence, we find that

$n\left(L_{\mathrm{x}}\right) \propto \frac{1}{L_{\mathrm{x}}} \frac{\mathrm{d} N}{\mathrm{~d} \log P} \frac{\mathrm{d} \log P}{\mathrm{~d} \log L_{\mathrm{x}}} \sim \frac{1}{L_{\mathrm{x}}} \frac{\mathrm{d} N}{\mathrm{~d} \log P}$.

This would match the observed shape of the LF of dim LMXBs if the factor $\mathrm{d} N / \mathrm{d} \log P$ were a constant. Unfortunately, it is difficult to construct this distribution from observations because of the many selection effects. Population synthesis calculations (e.g. Fragos et al. 2008) produce a variable distribution, which can only roughly be considered as constant. Observations suggest that initial orbital periods of binary stars do follow this dependence, i.e. $\mathrm{d} N / \mathrm{d} \log P=$ const. (Popova et al. 1982), and we assume this to hold approximately at all stages of binary evolution.

LMXBs with luminosities higher than $L_{\mathrm{x}}>10^{37.5} \mathrm{erg} / \mathrm{s}$ have predominantly giant companions (see above). The duration $\tau$ of the mass transfer (and hence the accretion stage) in these systems is significantly shorter than that for systems with main sequence donors, and to a large extent is determined by the mass transfer rate (see e.g. Webbink et al. 1983). We assume that the luminosity of LMXB is a power-law function of its orbital pe$\operatorname{riod} L_{\mathrm{x}} \propto P^{\alpha}$ (which is true for the magnetic stellar-wind braking dominated regime, $\alpha \sim 2.5-3.8$, see above, and also holds when nuclear evolution of the giant donor is responsible for its Roche lobe overflow, $\alpha \sim 1$, see Webbink et al. 1983). We also assume that the duration of the bright LMXB stage is inversely proportional to its luminosity $\tau \propto L_{\mathrm{x}}^{-1}$. This is approximately correct because the total mass, which might be accreted from a late-type giant donor stars does not vary much from system to system (see e.g. Webbink et al. 1983). Combining these, we obtain

$\frac{\mathrm{d} N}{\mathrm{~d} L_{\mathrm{x}}} \propto \frac{1}{L_{\mathrm{x}} P} \frac{\mathrm{d} P}{\mathrm{~d} L_{\mathrm{x}}} \propto \frac{1}{L_{\mathrm{x}}^{2}} \frac{\mathrm{d} \log P}{\mathrm{~d} \log L_{\mathrm{x}}} \approx L_{\mathrm{x}}^{-2}$.

Thus, almost independently of the mechanism that drives Roche lobe overflow inluminous LMXBs (with late-type giants), we obtain a slope of the LF similar to the observed one (Gilfanov 2004; Kim \& Fabbiano 2004). The condition for this to be correct is that the donor stars in these LMXBs are short-living giants. We can try to estimate the longest lifetimes of these giants assuming that the maximum mass, which might be accreted from them is $\$ 0.6 M_{\odot}$ (e.g. Webbink et al. 1983) and that their duty cycle is close to unity. In this case, $\tau_{\text {giants }} \lesssim$ $0.6 M_{\odot} /\left(2 \times 10^{-9} M_{\odot} /\right.$ year $) \sim 3 \times 10^{8}$ years. This means that the list of bright LMXBs in the Galaxy should alter after approximately $300 \mathrm{Myr}$.

We note here that the boundary between different types of donors at luminosities above and below $L_{\mathrm{x}} \sim 10^{37} \mathrm{erg} / \mathrm{s}$ was also previously noted in numerical simulations of LMXB populations in Fragos et al. (2008) and Kim et al. (2009), but that the slopes of the LMXB LF above and below this luminosity was not found to differ. The inability of particular numerical simulations to reproduce the observed break in the X-ray luminosity function of the LMXB population might indicate that we do not (yet) understand all details of the physical processes (e.g. the mode of the angular momentum loss, the common envelope phase, etc.) that shape the LMXB LF. We plan to perform more detailed calculations of the LMXB population in our future work.

Acknowledgements. This work is partially supported by RFBR grants 10-0200492-a, 10-02-00599-a, by Russian Federation President programm NSh5069.2010.2, Federal Russian Science Agency through the research contract 02.740.11.0575, and program of Presidium RAS P-19 and OFN-16.

\section{References}

Augusteijn, T., van der Hooft, F., de Jong, J. A., van Kerkwijk, M. H., \& van Paradijs, J. 1998, A\&A, 332, 561

Bandyopadhyay, R., Shahbaz, T., Charles, P. A., van Kerkwijk, M. H., \& Naylor, T. 1997, MNRAS, 285, 718

Bandyopadhyay, R. M., Shahbaz, T., Charles, P. A., \& Naylor, T. 1999, MNRAS, 306,417

Bandyopadhyay, R. M., Shahbaz, T., \& Charles, P. A. 2003, MNRAS, 340, L13 Belczynski, K., Kalogera, V., \& Bulik, T. 2002, ApJ, 572, 407

Bradshaw, C. F., Fomalont, E. B., \& Geldzahler, B. J. 1999, ApJ, 512, L121

Casares, J., Charles, P. A., \& Kuulkers, E. 1998, ApJ, 493, L39

Castro-Tirado, A. J., Brandt, S., \& Lund, N. 1992, IAUC, 5590

Chakrabarty, D. 1998, ApJ, 492, 342

Christian, D. J., \& Swank, J. H. 1997, ApJS, 109, 177

Cote, J., \& Pylyser, E. H. P. 1989, A\&A, 218, 131

Courvoisier, T. J.-L., Parmar, A. N., Peacock, A., \& Pakull, M. 1986, ApJ, 309, 265

Deloye, C. J. 2008, AIPC, 983, 501

Dubus, G., Lasota, J.-P., Hameury, J.-M., \& Charles, P. 1999, MNRAS, 303, 139 Ebisuzaki, T., Sugimoto, D., \& Hanawa, T. 1984, PASJ, 36, 551

Fender, R. P., Garrington, S. T., McKay, D. J., et al. 1999, MNRAS, 304, 865

Forman, W., Jones, C., Cominsky, L., et al. 1978, ApJS, 38, 357

Fragos, T., Kalogera, V., Belczynski, K., et al., 2008, ApJ, 683, 346

Gilfanov, M. 2004, MNRAS, 349, 146

Gottwald, M., Steinle, H., Pietsch, W., \& Graser, U. 1991, A\&AS, 89, 367

Ghosh, P., \& White, N. E. 2001, ApJ, 559, L97

Greiner, J., Cuby, J. G., McCaughrean, M. J., Castro-Tirado, A. J., \& Mennickent, R. E. 2001, A\&A, 373, L37

Grimm, H.-J., Gilfanov, M., \& Sunyaev, R. 2002, A\&A, 391, 923

Heger, A., Cumming, A., Galloway, D. K., \& Woosley, S. E. 2007, ApJ, 671, L141

Hinkle, K. H., Fekel, F. C., Joyce, R. R. et al. 2006, ApJ, 641, 479

Iben, I., Jr., \& Tutukov, A. V. 1984, ApJ, 284, 719

Jonker, P. G., Fender, R. P., Hambly, N. C., \& van der Klis, M. 2000, MNRAS, 315, L57

in't Zand, J. J. M., Cornelisse, R., \& Méndez, M. 2005, A\&A, 440, 287

Jonker, P. G., \& Nelemans, G. 2004, MNRAS, 354, 355

Jonker, P. G., Nelemans, G., \& Bassa, C. G. 2007, MNRAS, 374, 999

Kim, D.-W., Fabbiano, G. 2004, ApJ, 611, 846

Kim, D.-W., Fabbiano, G., Brassington, N. J., et al. 2009, ApJ, 703, 829

Krivonos, R., Revnivtsev, M., Lutovinov, et al. 2007, A\&A, 475, 775

Kulkarni, S. R., \& Narayan, R. 1988, ApJ, 335, 755 
M. Revnivtsev et al.: On the nature of break in the X-ray luminosity function of LMXBs

Kuulkers, E., \& van der Klis, M. 2000, A\&A, 356, L45

Kuulkers, E., den Hartog, P. R., in't Zand, J. J. M., et al. 2003, A\&A, 399, 663

Kuulkers, E., in't Zand, J. J. M., Atteia, J.-L., et al. 2010, A\&A, 514, A65

Liu, Q. Z., van Paradijs, J., \& van den Heuvel, E. P. J. 2007, A\&A, 469, 807

Markwardt, C. B., Swank, J. H., Marshall, F. E., \& in’t Zand, J. J. M 2000, in Rossi2000: Astrophysics with the Rossi X-ray Timing Explorer, March 22-24, at NASA's Goddard Space Flight Center, Greenbelt, MD, USA Marti, J., Mirabel, I. F., Rodriguez, L. F., \& Chaty, S. 1998, A\&A, 332, L45

Mason, K. O., \& Cordova, F. A. 1982, ApJ, 262, 253

Meshchryakov, A. V., Revnivtsev, M. G., Pavlinsky, M. N., Khamitov, I., \& Bikmaev, I. F. 2010, Astron. Lett. 36, 738

Naylor, T., \& Podsiadlowski, P. 1993, MNRAS, 262, 929

Nelemans, G., Portegies Zwart, S. F., Verbunt, F., \& Yungelson, L. R., 2001, A\&A, 368, 939

Paczynski, B., \& Sienkiewicz, R. 1981, ApJ, 248, L27

Patterson, J. 1984, ApJS, 54, 443

Popova, E. I., Tutukov, A. V., \& Yungelson, L. R. 1982, Ap\&SS, 88, 55

Postnov, K. A., \& Kuranov, A. G. 2005, AstL, 31, 7

Primini, F. A., Forman, W., \& Jones, C. 1993, ApJ, 410, 615

Pylyser, E., \& Savonije, G. J. 1988, A\&A, 191, 57

Rappaport, S., Verbunt, F., \& Joss, P. C. 1983, ApJ, 275, 713

Remillard, R. A., Levine, A. M., \& McClintock, J. E. 2009 [arXiv: 0903 . 3195]

Revnivtsev, M., Sazonov, S., Jahoda, K., \& Gilfanov, M. 2004, A\&A, 418, 927
Revnivtsev, M., Lutovinov, A., Churazov, E., et al. 2008, A\&A, 491, 209

Pfahl, E., Rappaport, S., \& Podsiadlowski, P. 2003, ApJ, 597, 1036

Ritter, H. 1999, MNRAS, 309, 360

Ritter, H., \& Kolb, U. 2003, A\&A, 404, 301

Sakano, M., Koyama, K., Murakami, H., Maeda, Y., \& Yamauchi, S. 2002, ApJS 138,19

Shahbaz, T., Watson, C. A., \& Hernandez-Peralta, H. 2007, MNRAS, 376, 1886 Sidoli, L., Belloni, T., \& Mereghetti, S. 2001, A\&A, 368, 835

Smale, A. P. 1998, ApJ, 498, L141

Smith, D. A., \& Dhillon, V. S. 1998, MNRAS, 301, 767

Sugizaki, M., Mitsuda, K., Kaneda, H., et al. 2001, ApJS, 134, 77

Taam, R. E. 1983, ApJ, 270, 694

Trinchieri, G., \& Fabbiano, G. 1991, ApJ, 382, 82

van der Sluys, M. V., Verbunt, F., \& Pols, O. R. 2005, A\&A, 431, 647

van Paradijs, J., \& White, N. 1995, ApJ, 447, L33

Verbunt F., \& Zwaan, C. 1981, A\&A, 100, L7

Voss, R., \& Gilfanov, M. 2006, A\&A, 447, 7

Voss, R., \& Gilfanov, M. 2007, A\&A, 468, 49

Voss, R., Gilfanov, M., Sivakoff, G. R., et al., 2009, ApJ, 701, 471

Wachter, S., \& Margon, B. 1996, AJ, 112, 2684

Webbink, R. F., Rappaport, S., \& Savonije, G. J. 1983, ApJ, 270, 678

White, N. E., \& Ghosh, P., 1998, ApJ, 504, L31

Xiang, J., Lee, J. C., \& Nowak, M. A. 2007, ApJ, 660, 1309 